\title{
Contribución al Desarrollo Social del Bachillerato en Línea como Estrategia de Acceso a la Educación Media Superior
}

\author{
Contribution to the Social Development of on Online \\ Baccalaureate Program as a Strategy for Access to Higher \\ Secondary Education
}

\author{
Yeily Delgado Cruz * \\ Edith J. Cisneros-Cohernour \\ Universidad Autónoma de Yucatán
}

\begin{abstract}
Este trabajo presenta los resultados preliminares de un estudio sobre un programa de Bachillerato en Línea en una universidad pública del sureste de México, el Bachillerato en Línea de la Universidad Autónoma de Yucatán; el cual surge ante la necesidad social de brindar mayor acceso a la Educación Media Superior. La investigación es cualitativa y se desarrolló dentro de un paradigma Interpretativista en la modalidad de estudio de casos; se centró en la percepción de los actores involucrados en el Proceso de Enseñanza Aprendizaje sobre las contribuciones de la institución al desarrollo social. La recogida de datos involucró análisis documental, y entrevistas semiestructuradas a los diferentes actores involucrados en el programa como son directivos de la institución y el programa, profesores del programa y estudiante del mismo. Los resultados preliminares indican que el bachillerato en línea enfrenta varios retos en el logro de su propósito, a pesar de los cuales contribuye al desarrollo social teniendo como principio la igualdad de acceso a la educación, logra formar alumnos en este nivel de enseñanza acorde a los niveles estandarizados de calidad y contribuye a su desarrollo humano, su situación económica y calidad de vida, de este modo satisface su perspectiva de bienestar subjetivo.
\end{abstract}

Descriptores: Acceso a la educación, Enseñanza secundaria superior, Aprendizaje en Línea, Educación superior.

\begin{abstract}
This paper presents the preliminary results of a study on an Online Baccalaureate program at a public university in Southeast Mexico. The research was focused on the perception of the actors involved in the Teaching Learning Process about the contributions of the institution to social development. Data collection involved documentary analysis, and semi-structured interviews. Preliminary results indicate that the online baccalaureate faces several challenges in achieving its purpose, in spite it contributes to social development, having as principle the equal access to education, managing to prepare students at this level of education according to the Standardized levels of quality thus contributing to their human development, economic status and quality of life, in order to satisfy their subjective well-being perspective.
\end{abstract}

Keywords: Access to education, Upper secondary education, Electronic learning, Higher education.

La investigación cuenta con el apoyo del Consejo Nacional de Ciencia y Tecnología (CONACTT). Es paste de un estudio nacional que cuenta con financiamiento del Programa de Desarrollo del Personal Académico (SEP).

*Contacto: yeilydc@gmail.com

ISSN: 0718-7378

www.rinace.net/rlei/
Recibido: 30/06/2017

$1^{a}$ Evaluación: 30/09/2017

Aceptado: $\quad 17 / 10 / 2017$ 


\section{Introducción}

La problemática del acceso a la educación ha sido abordada por organismos y organizaciones de diferentes niveles. En este sentido, es reconocido el importante papel que esta juega en cuanto al desarrollo en términos generales, reconociéndola como un derecho humano primordial que permite la inclusión y mejor desenvolvimiento en la esfera social.

El ámbito educativo no ha permanecido al margen de los adelantos científicos técnicos que se han desarrollado en la esfera social, haciendo uso de las Tecnologías de la Informática y las Comunicaciones en el Proceso de Enseñanza Aprendizaje. Estas tecnologías dado su rol protagónico en la formación general e integral de los individuos son de gran importancia en la formación del hombre nuevo.

Este trabajo presenta los resultados preliminares de un estudio sobre un programa de bachillerato en línea en una universidad pública del sureste de México con énfasis en el concepto de desarrollo social. La investigación es parte de la tesis del Doctorado Institucional en Ciencias Sociales de la Universidad Autónoma de Yucatán cuyo tema es "El bachillerato en línea como estrategia de acceso a la educación superior", la cual forma parte del proyecto: "Desarrollo y Validación de un Modelo de Evaluación de Competencias Docentes en Línea en Educación Superior”, auspiciado por las Redes Temáticas de Colaboración del Programa para el Desarrollo Profesional Docente (PRODEP), de la Dirección General de Educación Superior Universitaria de la Secretaría de Educación Pública de México.

\section{Marco teórico}

A continuación, se examinan los principales conceptos a trabajar en esta investigación: desarrollo social y equidad e inclusión educativa. Identificados a partir del propósito del BEL de brindar una educación de calidad a distancia mediada por el uso de las TIC y reducir de forma significativa las brechas sociales, económicas y culturales persistentes en la sociedad, por lo que debe contribuir de este modo al desarrollo social mediante el acceso equitativo e inclusivo a la EMS (UADY, 2012a).

En el período de los años 50 y 60 el concepto de desarrollo se vio influenciado por los fuertes criterios económicos de la época; hacia la siguiente década, se aparta un poco de esta concepción concibiendo elementos como la pobreza, la desigualdad, el desempleo, el acceso a servicios educativos, entre otros; procurando que esta forma de pensarlo se ajuste más a los criterios de validez internacionales, teniendo en cuenta para ello cuestiones naturales de cada país. Los cuales podrían analizarse desde los derechos humanos, económicos y sociales. En esta concepción del concepto de desarrollo incluye el análisis la desigualdad de acceso a los bienes sociales, tales como la educación.

En este sentido, los diversos análisis apuntaron hacia la relación entre la educación y la economía, sobre todo a partir de la aparición de la teoría de desarrollo de capital humano, donde independientemente de la divergencia de criterios en este sentido y de que no se ha podido evaluar con exactitud este impacto, coincidían en las positivas influencias de la educación en el desarrollo. Lo cual no significa una relación directa pues ya se ha reconocido el carácter necesario de la educación para el logro del desarrollo a la vez que 
se identifica que no es suficiente para el alcance del mismo, reconociendo la existencia de otros elementos a considerar (De Puelles y Torreblanca, 1995).

De este modo, el concepto de desarrollo social se ha caracterizado por el reconocimiento de las capacidades humanas para alcanzar el desarrollo, la necesidad de alcanzar sus libertades en este sentido que conlleven al bienestar de los mismos. Referente al desarrollo social teniendo en cuenta las capacidades, organismos como la UNESCO han reconocido la necesidad de entender las capacidades de los individuos, ya sea individuales o colectivas para construir su desarrollo, enfatizando la necesidad de centrar, en este sentido, la atención en el desarrollo humano atendiendo a las necesidades específicas de cada sociedad, reconoce la inexistencia de una relación directa entre educación y desarrollo social, por lo cual demanda ocuparse de las necesidades de las personas desde un enfoque teórico como es el enfoque de las capacidades (UNESCO, 1999).

Entre los investigadores en este aspecto teórico destacan Amartya Kumar Sen y Martha Craven Nussbaum. Para Sen (1988), el éxito del desarrollo tiene que ser juzgado en términos de lo que mejora las condiciones de vida, por lo que el vínculo entre desarrollo económico y crecimiento económico es una cuestión de importancia, y a su vez una fuente de confusión; pues desarrollo económico no puede abstraerse del suministro de alimentos, ropa, vivienda, servicios médicos, instalaciones educativas, etc., y transformar la estructura productiva de la economía, son asuntos de crecimiento económico. Al establecer una distinción entre desarrollo y crecimiento, distingue diferentes fuentes de contraste.

- En la medida en que el crecimiento económico se refiere sólo al Producto Nacional Bruto (PNB) per cápita, deja de lado la cuestión de la distribución de ese PNB entre la población.

- El PNB captura únicamente los medios de bienestar que se transan en el mercado, dejando fuera los beneficios y costos que no tienen un precio asociado a ellos.

- La valoración de los productos básicos en el PNB reflejará los sesgos que puedan tener los mercados.

- El ingreso real que goza una persona en un año determinado refleja el grado de bienestar de esa persona en ese tiempo; evaluar el tipo de vida de la persona, debemos tener una visión más integral de su vida.

- El PNB es, una medida de la cantidad de medios de bienestar que tiene la gente, y no nos dice cuáles son las personas involucradas que consiguen salir de estos medios (Sen, 1988).

Sen (2000), reconoce la importancia de la libertad para el proceso de desarrollo en dos sentidos; en primera instancia la de la evaluación, donde el progreso estaría dado por el aumento o no que se haya producido en las libertades de las personas; y en segunda instancia la de la eficiencia, identificando la dependencia del desarrollo a la libre agencia de las personas. La importancia de la libertad para el logro del desarrollo social puede argumentarse desde las libertades fundamentales como lo es la educación, la cual contribuye eficientemente al desarrollo humano y progreso económico, evidencia el rol que desempeñan diversas instituciones, como por ejemplo el sistema educativo, y los valores sociales que influyen en las libertades de las personas, vistas como oportunidades sociales, como lo es una educación inclusiva, que propicia la participación económica de las personas, permitiéndoles construir su realidad como entes activos brindándoles la libertad de elegir (Sen, 2000). 
Según Nussbaum (2013), es necesario centrarse en las historias de las personas en cada contexto, dado que tienen innumerables en aras de alcanzar una vida digna y justa. Cuando se refiere a vida digna hace énfasis en una calidad de vida cualitativamente contextual, donde para algunos puede esta entenderse como integridad física y para otros como el acceso a servicios públicos tales como la educación, etc. El enfoque no se centra en el bienestar total o medio sino que se centra en cada persona, en su libertad, en una concepción de justicia social y de derecho constitucional, dado que para alcanzar dicha justicia social se requiere garantizar a los miembros de la sociedad una vida a la altura de la dignidad humana. En este sentido, se concentra en la diversidad cultural atendiendo a las capacidades dadas por la dignidad humana, donde el gobierno juega un papel importante en el desarrollo de estas capacidades así como el ejercicio pleno de la libertad. Identifica entre otros retos y limitaciones contemporáneas del enfoque el acceso a la educación (Nussbaum, 2013).

Nussbaum (2002), plantea las siguientes diez capacidades que considera necesarias para el desarrollo humano, aunque plantea que no son las únicas y que la existencia de otras es un proceso contextual:

- Vida: Vivir una vida humana de extensión normal; no morir prematuramente, o antes de que se haya reducido de modo que no merezca la pena vivirse.

- Salud corporal: Tener buena salud de forma general en todos sus aspectos.

- Integridad corporal: Poder moverse libremente, capaces de seguridad ante cualquier tipo de asalto y violencia; tener oportunidades para la satisfacción sexual y elección reproductiva.

- Sentidos, imaginación y pensamiento: Utilizar los sentidos, de imaginar, pensar y razonar de forma libre.

- Emociones: Vincularse con cosas y personas fuera de uno mismo de forma emotiva y libre de temores y preocupaciones.

- Razón práctica: Plasmar una concepción del bien y de comprometerse en una reflexión crítica acerca del planteamiento de la propia vida.

- Afiliación: Vivir con y hacia otros, preocupándose por otros seres humanos por medio de la interacción social.

- Otras especies: Cuidar los animales, las plantas y el mundo de la naturaleza y en relación con todo ello.

- Juego: Reír, jugar y disfrutar de actividades recreativas.

- Control del propio entorno: En lo político participar en elecciones políticas de forma libre. En el aspecto material ser poseedor de materiales en términos de oportunidades reales (Boni Aristizábal, 2005).

$\mathrm{Al}$ aumentar la educación, aumenta la eficiencia de una persona en la producción de bienes, por lo que estamos en presencia de desarrollo de capital humano, lo cual de igual modo puede aumentar el valor productivo del mismo así como su valor de renta, y en consecuencia conducir al desarrollo económico y por tanto aumentar la capacidad económica en términos adquisitivos de la persona; lo anterior aunado a que la capacidad de lectura e interpretación de la persona con alto nivel de renta le permite ser tomada en cuenta por los demás, aumenta su nivel de libertad, lo cual se ve reflejado en su bienestar. 
Como hemos podido apreciar, tanto Sen como Nussbaum, en el momento de abordar sobre el desarrollo social no pierden de vista la influencia que ejerce en el bienestar de las personas. En el análisis del desarrollo social en cuanto a bienestar, encontramos autores como Consuelo Uribe (2004) para quien la mejoría en índices de bienestar, tales como el acceso a educación, sería lo que conduce al desarrollo social, entendiendo que este es construido. Este autor hace un llamado a la política social a dar seguimiento al acceso de sus ciudadanos a los servicios sociales en aras de asumir su responsabilidad social de igualdad y equidad social.

Hacia la búsqueda del entendimiento y caracterización del bienestar podemos apreciar que en relación al individuo de forma contextual Várguez (2015) se refiere al bienestar subjetivo como un proceso de construcción que realiza la persona dada su interpretación del mundo que le rodea, el cual es constante dadas las diferentes situaciones a las que puede afrontarse. En este sentido, plantea 4 características:

Su totalidad, atendiendo a los aspectos en los que la persona centra su bienestar.

- Su relatividad, dada la variación que se presenta en las formas en que cada persona construye su bienestar.

- Su desigualdad, presentada por la diversidad característica de personas y entornos en que es construido el bienestar.

- Su dinamismo, puesto que la persona puede cambiar su perspectiva atendiendo a sus experiencias de vida (Várguez, 2015).

Atendiendo a todo lo planteado hasta el momento es posible entender el concepto de desarrollo social como un proceso dinámico y contextual que es construido por las personas dadas sus capacidades; donde se conjugan sus percepciones de libertad y bienestar subjetivo.se centra en el desarrollo humano como uno de los elementos que contribuyen al desarrollo económico.

Otros conceptos fundamentales son los de equidad e inclusión educativa en relación con el desarrollo social. En el plano educativo, a partir de los años 90, las discusiones han sido objeto de análisis internacional; aumentando la conciencia de su importante papel para el desarrollo económico y social. En este sentido el Comité de Derechos Económicos, Sociales y Culturales reconoce que el medio principal para que las personas marginadas social y económicamente, independientemente de su edad, puedan participar completamente en sus comunidades es la educación (UNESCO, 2008).

En el año 2000, la evaluación de la Educación para Todos evidenció que existen más de 113 millones de niños sin acceso a la enseñanza primaria y 880 millones de adultos analfabetos en el mundo. Se encontró que la discriminación entre los géneros continúa presente en los sistemas de educación y que la calidad del aprendizaje y la adquisición de valores humanos y competencias distan de las aspiraciones y necesidades de los individuos y las sociedades; que se niega a jóvenes y adultos el acceso a las técnicas y conocimientos necesarios para encontrar empleo remunerado y participar plenamente en la sociedad. Los resultados de esta evaluación enfatizan que, si no se avanza rápidamente hacia la educación para todos, no se lograrán los objetivos de reducción de la pobreza, adoptados en el plano nacional e internacional, y se acentuarán aún más las desigualdades entre países y dentro de una misma sociedad (UNESCO, 2000). 
Diferentes documentos como: La Declaración Mundial sobre Educación para Todos y el Marco de Acción para Satisfacer las Necesidades Básicas de Aprendizaje: Directrices para la aplicación de la Declaración Mundial sobre Educación para Todos, aprobadas por la Conferencia Mundial sobre Educación para Todos en Jomtien (1990); Higher Education: The Lessons of Experience(1990); Educación y Conocimiento: Eje de la Transformación Productiva con Equidad y sus documentos de política en educación superior (1995); La Declaración de Recife de los Países del Grupo E-9, aprobada por la reunión ministerial de examen de los países del Grupo E-9 en Recife (Brasil) (2000); El Marco de Acción de Dakar: Educación para Todos: cumplir nuestros compromisos comunes, aprobado en el Foro Mundial de Educación de Dakar (Senegal) (2000); coinciden en la importancia de la construcción de una política consensuada en relación a la educación, de una reforma autónoma y descentralizada en las instituciones de educación superior y en la accesibilidad de una educación de calidad para todos en especial las poblaciones de mayor vulnerabilidad, dado que ante la mundialización de la educación los países y hogares que no tengan acceso a ella estarán propensos a una mayor marginalización en una economía internacional cada vez más próspera (Banco Mundial, 1994; Kent, 1995; UNESCO, 2000, 2017).

Así mismo, la Declaración de Incheon y el Marco de Acción para la realización del Objetivo de Desarrollo Sostenible 4 propone "Garantizar una educación inclusiva y equitativa de calidad y promover oportunidades de aprendizaje permanente para todos" (UNESCO, 2015, p. 7) concentra los esfuerzos en el acceso, la equidad, la inclusión, la calidad y los resultados del aprendizaje, dentro de un enfoque del aprendizaje a lo largo de toda la vida, como piedra angular para una educación transformadora, para lo que considera preciso aprovechar las TIC para reforzar los sistemas educativos, la difusión de conocimientos, el acceso a la información, el aprendizaje efectivo y de calidad, y una prestación más eficaz de servicios, en este sentido se plantea:

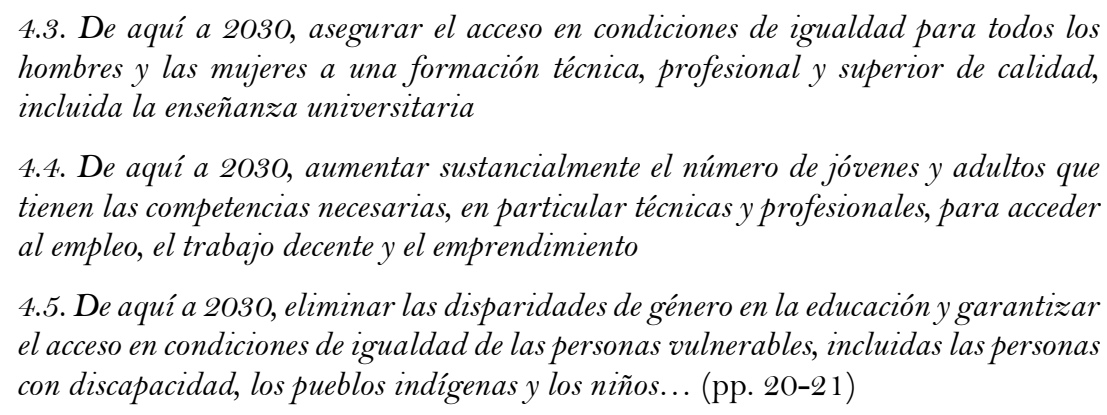

En América Latina el desafío es asegurar el acceso a la educación de calidad a toda la población, enfatiza la atención a las poblaciones en situaciones de vulnerabilidad. Esto platea la necesidad de una sólida voluntad política y una mejora correspondiente de la calidad, junto con un cuerpo docente compuesto de profesores capacitados y motivados, así como medidas orientadas a alcanzar a los grupos de población marginados o excluidos (UNESCO, 2000).

Esta situación se presenta especialmente en Educación Media Superior (EMS), entendida como un nivel de enseñanza que es cursado una vez culminada la Secundaria y previo al ingreso a la Educación Superior, es denominado de igual modo como "Preparatoria", "Bachillerato" o "Segundo nivel de secundaria" según sea la región del mundo. Es un nivel educativo que, de acuerdo con los parámetros de OCDE, en 2014, tuvo una tasa de estudiantes matriculados entre los 15-19 años de edad se sitúan entre el 80\% y el $90 \%$ en 
la mayor parte de sus países miembros. Sin embargo, existen diferencias entre los países, como es el caso de México (56\%) e Irlanda (95\%). La tasa de escolarización promedio se encuentra entre el $55 \%$ y el $65 \%$, donde Italia alcanza la tasa más alta, con un $75 \%$ y México la más baja, con un 38\% (MECD, 2016, p. 15).

En el caso de México, el Plan de Desarrollo Nacional declara como uno de sus ejes prioritarios la Educación de Calidad; en el cual se considera como segundo objetivo: Garantizar la inclusión y la equidad en el Sistema Educativo. Para esto, la estrategia consiste en ampliar las oportunidades de acceso a la educación en todas las regiones y sectores de la población. Esta estrategia tiene especial importancia en el nivel de EMS. De acuerdo con el Consejo Nacional de Evaluación de la Política de Desarrollo Social (CONEVAL), considera como dos de los principales retos de la EMS y Educación Superior en México la cobertura insuficiente y la desigualdad en el acceso. En el año 2010, el nivel de cobertura de EMS fue de $66 \%$ y $28 \%$ en educación superior (ES). La eficiencia terminal de $58 \%$ para la EMS y $63 \%$ para ES. Si tomamos en cuenta que la educación se financia principalmente con recursos públicos, es posible concluir que existe falta de equidad en el acceso y de subsidios que podrían considerarse regresivos. De igual modo, la calidad es un gran reto difícil de medir, por lo que el Gobierno Federal ha optado por utilizar retos, metas y avances de la calidad educativa básicamente a través de indicadores indirectos, como son los registros o las acreditaciones (CONEVAL, 2014; UADY, 2014).

Un elemento importante a considerar es que a nivel internacional se ha propuesto que la Educación a Distancia (EAD), pueda dar respuesta a la necesidad social existente de proveer acceso a la educación. A raíz de esto, un comité de expertos en diseño normativo realizó un diagnóstico de la situación de la EAD en México, y propuso las medidas para su desarrollo. Se encontró que existe diversidad en las diferentes dimensiones (conceptualización, normatividad, organización y oferta de programas) de esta modalidad y que no se cuenta con una regulación que sustente y respalde la EAD con criterios de calidad, pertinencia y equidad, lo cual limita su desarrollo y provoca la pérdida de oportunidades para el acceso a la educación superior y la ampliación de la cobertura; identifica que gran parte de la oferta en esta modalidad atiende necesidades particulares, y no aquellas que surgen en el sector productivo o social. Por tanto, se identifica para la EAD pueda promover el acceso a la educación, es necesario examinar los retos que enfrenta en relación con abatir las desigualdades territoriales, así como atender a los grupos en situación de desventaja, dándole la misma importancia a la modalidad tradicional que a la EAD. Otro reto es la necesidad de promover la coordinación de las autoridades educativas y las Instituciones de Educación Superior (IES), para la regulación de esta modalidad de estudio y asegurar los recursos tecnológicos y humanos.

La problemática de la EMS es especialmente seria en el sureste de México. En el caso de Yucatán, se observa que en el período 2008 y 2010, el estado es clasificado con un alto grado de rezago social, ocupando la posición número 9 en el país. En 2010, el porcentaje de la población de 16 años o más nacida hasta 1981, tenía un rezago educativo del 35.6\%. Esta problemática, constituye uno de los principales retos en materia de política social; por lo que en el Plan Estatal de Desarrollo 2012-2018, el Gobierno del Estado de Yucatán, identifica entre los 5 grandes temas prioritarios el de proveer una educación de calidad (UADY, 2014).

Aunque hasta la fecha se ha avanzado en brindar mayor acceso a este nivel de educación, en Yucatán aún existe un número considerable de personas que no logran ingresar a este 
nivel educativo. Según datos del Instituto Nacional de Estadística y Geografía (INEGI) en 2010 , sólo el $63 \%$ de la población yucateca de 15 y más años tiene algún grado aprobado en educación básica y el 8.2\% es el grado promedio de escolaridad de la población de 15 y más años en el 2010. En el 2013 de una demanda de ingreso de 4204 fueron aceptados a la EMS 3069, lo que representa el 73\% (UADY, 2014).

En el caso de la Universidad Autónoma de Yucatán (UADY), en el ciclo escolar 2014-2015 el índice de abandono escolar en el bachillerato fue del $17.4 \%$ en el estado, un $2.6 \%$ por encima de la del país. Atendiendo a la proyección de cobertura escolar en el nivel de bachillerado, el Estado de Yucatán espera, para el curso escolar 2020-2021, que aún persista un $14 \%$ de la población entre 15 y 17 años de edad no atendida. Identifica como principal reto establecer una sola medida que rija a la diversidad de Instituciones de Educación Superior que registra el país, considerando las personas que por diferentes factores no se encuentran en las condiciones para cursar su educación en la modalidad presencial (UADY, 2013).

Para enfrentar con éxito y eficacia las demandas sociales del estado de Yucatán y del contexto de la educación superior la UADY plantea en su Plan de Desarrollo Institucional (PDI), mejorar significativamente sus estándares de desempeño para responder con mayor calidad y oportunidad a las variadas y complejas demandas del desarrollo social y económico del Estado de Yucatán y atender con éxito los retos que toda institución de educación superior en México y en el mundo enfrenta reforzando de este modo los esfuerzos institucionales para hacer realidad la visión de la UADY para el 2022 a través de nuevas políticas y estrategias establecidas y la aplicación del Programa Integrador Responsabilidad Social Universitaria, el cual está integrado ahora por 12 programas prioritarios, entre los cuales se encuentra la ampliación y diversificación de la oferta educativa y el aseguramiento de la pertinencia y calidad de los programas de bachillerato, licenciatura y posgrado (UADY, 2014).

Como respuesta al Plan Nacional de Desarrollo 2007-20012, la UADY (2013a) ha planteado "impulsar el desarrollo y utilización de nuevas tecnologías en el sistema educativo para apoyar la inserción de los estudiantes en la sociedad del conocimiento y ampliar sus capacidades para la vida", y que "ampliar la cobertura, favorecer la equidad y mejorar la calidad y pertinencia de la educación superior”, para lo cual la institución decidió utilizar como estrategia para alcanzar la cobertura, por medio de modalidades educativas no convencionales, especialmente por medio de la enseñanza a distancia.

En consistencia con lo anterior, la UADY creó el Bachillerato en Línea (BEL) en el año 2012, el cual se fundamenta en los ejes, objetivos y estrategias planteados por el Gobierno de la República Mexicana, en el Plan Nacional de Desarrollo 2007-2012; el Gobierno del Estado de Yucatán, en el Plan Estatal de Desarrollo 2007-2012 y en el Programa de Desarrollo Institucional 2010-2020 de la Universidad Autónoma de Yucatán (UADY, 2012a).

\section{El Bachillerato en Línea de la Universidad Autónoma de Yucatán}

El Bachillerato en Línea (BEL), busca reducir significativamente las brechas sociales, económicas y culturales persistentes en la sociedad brindando una Educación de calidad a Distancia mediada por el uso de las Tecnologías de la informática y las Comunicaciones planteándose como objetivo "Formar integralmente bachilleres, mediante la implementación de herramientas tecnológicas, en los ámbitos académico, personal, social 
y cultural que además les proporcione la posibilidad de incorporarse al nivel licenciatura" (UADY, 2012a, p. 14). El cual se encuentra dirigido a personas que tuvieran concluido sus estudios de secundaria con una edad mínima 18 años cumplidos.

El BEL es un programa de estudio que surgió como respuesta al Plan Nacional de Desarrollo 2007-20012, por lo que la universidad se planteó "impulsar el desarrollo y utilización de nuevas tecnologías en el sistema educativo para apoyar la inserción de los estudiantes en la sociedad del conocimiento y ampliar sus capacidades para la vida”, así como "ampliar la cobertura, favorecer la equidad y mejorar la calidad y pertinencia de la educación superior" (UADY, 2013, p. 5), para lo cual la institución decidió utilizar como estrategia para alcanzar la cobertura, por medio de modalidades educativas no convencionales, especialmente por medio de la enseñanza a distancia.

En consistencia con lo anterior, la UADY, en conjunto con la Dirección General de Desarrollo Académico y la Coordinación del Sistema de Educación Media Superior creó el BEL en el año 2012, el cual se fundamenta en los ejes, objetivos y estrategias planteados por el Gobierno de la República Mexicana, en el Plan Nacional de Desarrollo 2007-2012; el Gobierno del Estado de Yucatán, en el Plan Estatal de Desarrollo 2007-2012 y en el Programa de Desarrollo Institucional 2010-2020 de la UADY (2012a).

El BEL busca reducir significativamente las brechas sociales, económicas y culturales persistentes en la sociedad brindando una Educación de calidad a Distancia mediada por el uso de las TIC, se plantea como objetivo: "formar integralmente bachilleres, mediante la implementación de herramientas tecnológicas, en los ámbitos académico, personal, social y cultural que además les proporcione la posibilidad de incorporarse al nivel superior e insertarse a un campo laboral" (UADY, 2012a, p. 14).

Este programa, en sus inicios estaba dirigido a personas mayores de 18 años pero en la actualidad se encuentra dirigido a personas que hayan concluido sus estudios de secundaria y deseen retomar o continuar sus estudios de nivel bachillerato, maneja una estructura de plan de estudios con una duración de 3 años, el cual es desarrollado bajo una modalidad en línea, estructurada en seis niveles; en total, el alumno debe cursar 48 asignaturas, de los cuales 39 son básicas y nueve optativas, se espera que invierta un promedio de cuatro horas diarias, cinco días a la semana, aunque la duración del programa depende totalmente del estudiante, y particularmente de su disponibilidad de tiempo y habilidad tecnológica; cada nivel equivale a un semestre en el bachillerato presencial, y cada uno posee ocho asignaturas; para concluir en el tiempo necesario es preciso que el estudiante cargue al menos cuatro asignaturas por periodo, sin embargo esto es ajustable a sus necesidades.

En cuanto al personal de gestión, administrativo, de orientación y docente, este se encuentra conformado por: un Coordinador del programa, un Tutor Orientador, cuya función consiste en brindar seguimiento a los estudiantes del BEL, aconsejar sobre decisiones académicas, apoyo en conflictos que pudieran estar afectando el desempeño de los estudiantes en el programa, un Responsable de Gestión de Asesores, quien se encarga de coordinar el trabajo de los asesores en las diferentes asignaturas, y un Responsable de la Plataforma, quien se encarga de resolver dudas respecto al uso de la plataforma, brindar apoyo a los estudiantes y tutores en relación con las diferentes herramientas. Sus profesores en la actualidad son 11 . 
En general, se puede constatar que son escasas las investigaciones, aunque han cobrado auge en la última década sobre el tema de equidad e inclusión en programas de bachillerato y especialmente en línea como estrategia para el desarrollo social. Las existentes analizan las políticas para alcanzar la equidad e inclusión educativa en la enseñanza tradicional, no así las nuevas tendencias de la educación a distancia mediada por tecnología. Son objeto de análisis diferentes niveles de enseñanza donde la EMS es la menos estudiada. En los estudios existentes se evidencia el desequilibrio y la incompatibilidad entre las expectativas y las opciones educativas; la accesibilidad y la calidad a la educación como un reto y que el modelo aplicado es homogéneo, no ajustado a las necesidades (Bracho, 2002; CONEVAL, 2014; MECD, 2016; OCDE, 2015; Ordorika, 2014).

Respecto a las políticas para la implementación de las tecnologías de la informática y las comunicaciones en espacios educativos, así como su calidad, evaluación y acreditación, de igual modo son escasas las investigaciones, la mayor parte son en Licenciatura o Educación Superior mientras que el Bachillerato ha sido el menos investigado, a pesar de ser un nivel en el que la demanda actual es alta y los estudiantes cuentan con un desarrollo cognitivo suficiente para estudiarlo (ANUIES, 2015).

\section{Metodología}

Esta investigación es cualitativa y se desarrolla dentro de un paradigma Interpretativista, cuya meta es interpretar la vida social y entender el mundo complejo de la experiencia de vida, desde el punto de vista de aquellos que la viven con una perspectiva cultural e histórica, con la intención de entender e interpretar el significado atribuido por los sujetos a sus acciones en una realidad socialmente construida (Moreira, 2002; Sandín, 2003; Schwandt, 1994).

El estudio se llevó a cabo en la modalidad de estudio de casos. Como afirma Creswell (2009), un estudio de caso es un estudio acerca de un objeto definido que involucra la exploración detallada a lo largo de un período de tiempo, lo suficientemente extenso, que permita el entendimiento profundo del objeto de estudio y del contexto en que éste se ubica por medio de métodos múltiples de recolección de datos y múltiples fuentes de información altamente contextualizadas.

Atendiendo a Stake (2007), el caso es algo específico y complejo en funcionamiento, el cual puede estar constituido por una persona, un grupo de personas o un programa. Cuando el interés está dado porque con su estudio aprendemos sobre otra cosa, es decir, analizamos algo diferente que la comprensión de lo que estudiamos estamos en presencia de un estudio de casos instrumental. El estudio de caso en esta investigación es el Bachillerato en Línea de la UADY para lo cual partimos del estudio del caso instrumental de Omar, quien es un estudiante de éxito del BEL.

El BEL fue seleccionado para esta investigación porque es el único programa de enseñanza en línea con que cuenta la universidad, y constituye la base para el desarrollo e implementación de futuros programas de estas características en la universidad. Asimismo, porque tras 5 años de implementación, no ha sido objeto de investigaciones en cuanto a sus fortalezas y debilidades en el cumplimiento de su misión de facilitar el acceso a la EMS. La selección del estudiante estuvo dada porque es un caso de éxito del BEL, aglutina varias de las características que posee la población estudiantil de dicho programa, tiene dificultades visuales que le afectó su desarrollo en la enseñanza tradicional e intentó 
cursar estudios de bachiller en varias instituciones que no lo aceptaron por su edad, es el mayor de cinco hermanos y responsable junto a su mamá de la economía del hogar por lo que debió además conjugar el estudio con el trabajo.

Las técnicas empleadas en la recolección de datos involucraron análisis documental y entrevistas semiestructuradas como estrategias de recogida de datos. El análisis documental se llevó a cabo con base en la información proporcionada por el programa, incluyendo sus antecedentes y características, lo cual contribuyó a una mayor compresión del programa y su contexto. Las entrevistas se llevaron a cabo con la coordinadora del programa de Bachillerato en Línea, con el Director de Desarrollo Académico de la Universidad y el Director de Responsabilidad Social Universitaria. Se realizaron de igual modo entrevistas a docentes del BEL y a un estudiante graduado del programa que ingresó al mismo con más de 18 años y a su mamá.

Para realizar las entrevistas semiestructuradas se elaboró un protocolo de entrevistas con preguntas abiertas, brindándole a los entrevistados una copia de estas y considerando que el investigador tiene la libertad de incluir preguntas para precisar conceptos u obtener mayor información. Para ello las entrevistas tuvieron una duración máxima de una hora y media en la cual el investigador permaneció alerta en aras de reconocer información importante y explorar para obtener más detalles, a la vez que se tomaron notas de las mismas y se grabó la conversación previa autorización verbal de los entrevistados (Badilla, 2006; Creswell, 2009; Hernández Sampieri y Baptista Lucio, 2014; Ruiz Olabuenaga, 2003; Sandín, 2003; Sandoval Casilimas, 1996; Stake, 2007). Estas, se realizaron con el Director General de Desarrollo Académico, el Director General de Responsabilidad Social Universitaria, la coordinadora del BEL, profesores y directivos del BEL, el estudiante seleccionado del BEL, así como su mamá.

La observación no participante permitió describir los diferentes contextos en los que se desarrolla la investigación, lo cual permite al lector una mejor comprensión de cada escenario estudiado, lo cual reduce el sesgo del investigador ya que los participantes desconocen en qué momento están siendo estudiados (Badilla, 2006; Moreira, 2002; Sandín, 2003; Stake, 2007). Se observaron los procesos que se desarrollan en el BEL, así como el contexto del mismo y en el que se desenvuelve el estudiante seleccionado.

El análisis documental se realizó con el propósito de conocer las características tanto de la universidad como del programa, involucró la revisión de diferentes documentos institucionales, entre los que se encuentran: El Modelo Educativo de Formación Integral, El Plan de Desarrollo Institucional, Reglamento Interior de las escuelas preparatorias, Estatuto General de la Universidad Autónoma de Yucatán, Reglamento del personal académico, Plan de Desarrollo UADY Virtual, el Programa Educativo de Tipo Medio Superior. Bachillerato en Línea, Segundo informe de la gestión 2015-2018 de la UADY, así como la documentación utilizada en los procesos de enseñanza en línea en el programa de Bachillerato en Línea de la UADY (Sandín, 2003; UADY, 2002a, 2002b, 2011, 2012a, 2012b, 2013, 2014, 2016).

Para el análisis de datos fueron grabadas, previa aprobación de los participantes, todas las entrevistas, las cuales fueron transcritas. Durante el proceso se incluyeron reflexiones y comentarios acerca de la información obtenida. Para realizar el análisis de la información obtenida por medio de las entrevistas semiestructuradas se utilizó el diagrama de afinidad. Este diagrama fue diseñado por Jiro Kawakita, que permite categorizar e integrar los datos 
que tienen una afinidad mutua (Hirata, 2003). El empleo del software Atlas Ti permitió categorizar y realizar diagramas.

El uso de diferentes fuentes de información, así como la triangulación de los datos, contribuye a una mejor comprensión de la información que se obtuvo en el proceso en cuanto a las transformaciones que se producen en los estudiantes que cursan el BEL en cuanto a desarrollo social y las acciones que desarrollan para enfrentar los retos que en su actuar cotidiano afrontan en este sentido (Stake, 2007).

\section{Resultados}

A continuación, se presentan los resultados del estudio con base en la teoría de las capacidades de Nussbaum, contrastando las diez capacidades de esta teoría con las contribuciones del BEL al desarrollo social, atendiendo a lo encontrado dadas las perspectivas de los actores en su experiencia en el desarrollo del programa.

\subsection{Directivos}

Los directivos identificaron cinco aspectos en los que contribuye a su criterio el BEL al desarrollo social: rehabilitación social, innovación educativa, ingreso, flexibilidad y transformación. En este apartado discutiremos las que no fueron identificadas por otros actores.

Los directivos, manifiestan que la enseñanza en línea constituye una transformación en cuanto a modo de concebir y desarrollar la enseñanza, la cual debe adecuarse a las necesidades contextuales de las personas de modo que facilite el acceso de aquellas que por diferentes razones se les dificulta la enseñanza presencial, evidenciando de este modo el cumplimiento de la capacidad que Nussbaum llama "afiliación", mediante la cual el individuo debe ser tratado como igual sin discriminación y por tanto se han de concebir programas en consecuencia con ello, tal como

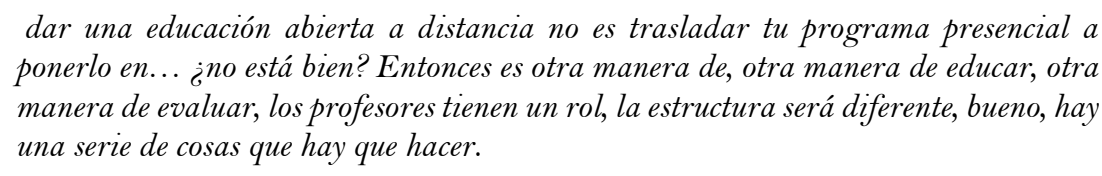

Del mismo modo, se evidencia las aportaciones del BEL en esta capacidad en cuanto al ingreso al programa, dado que ha modificado su modo de ingreso, en sus inicios por medio de la realización del EXANI como habitualmente lo hacen otras instituciones, en la actualidad se le realiza un examen diagnóstico a los aspirantes con la intención de conocer sus habilidades tecnológicas, no contempla la necesidad de un promedio para poder ingresar al mismo, el requisito es haber concluido la secundaria, lo cual, a criterio de los directivos permite un mayor desarrollo dado que brindas mayor oportunidad de estudio a todo el que lo desee y requiera, lo anterior se evidencia cuando plantean:

$$
\begin{aligned}
& \text { imaginemos que hoy todos entran por promedio, porque ya dije porque es un } \\
& \text { estandarizado, entonces el que no cumple el promedio no entra. Entonces yo ya tome } \\
& \text { un criterio que para algunos van a decir: con esto ya hiciste que no entren muchos de } \\
& \text { los que podían entrar. Si creemos que si tienen grandes capacidades, pero no tienen } \\
& \text { oportunidades, entonces ¿̇cómo le brindo la oportunidad de demostrar que si puede? }
\end{aligned}
$$

Ejemplifican la realización de convenios que permiten la superación a jóvenes de forma tal que les facilite la integración a la sociedad y poder incidir de este modo de forma positiva en su desarrollo, un ejemplo de ello lo constituye: 
tenemos un convenio con el centro, el CEAMA, ahorita no recuerdo exactamente cuál es el... pero es el de... es un centro especializado para la atención de menores que han tenido algún problema con la justicia. Entonces se firma el convenio para que ellos puedan llevar el bachillerato en línea en este centro. Entonces bueno pues para hacer una rehabilitación, una readaptación social.

\title{
3.2. Docentes
}

Los docentes identificaron como aspectos en los que contribuye a su criterio el BEL al desarrollo social la innovación educativa, la atención diferenciada, la calidad de vida y la flexibilidad. En este apartado discutiremos los dos que no fueron identificadas por otros actores.

Para los docentes la enseñanza en línea constituye una motivación dado el interés que poseen hoy las jóvenes en las tecnologías para elevar su nivel de preparación académica y de este modo su calidad de vida, permitiéndoles aspirar a un mejor puesto laboral, evidenciándose de este modo el alcance de las capacidades de "vida", de "salud corporal", "sentidos, imaginación y pensamiento" y "control del propio entorno" de Nussbaum dado que, en las propias palabras de los actores involucrados:

\begin{abstract}
La tecnología ahorita pues está en todos lados ¿no? con programas gubernamentales ya hay internet gratuito en muchos lugares, parques, escuelas, bibliotecas comunitarias y esto ayuda a que, aprovechar que el estudiante, el muchacho o el adulto está pegado a la tecnología a través incluso de su celular para poder acceder a la educación no solamente que sea un motivo de entretenimiento, una herramienta de entretenimiento si no aprovechar esa conexión que ya tiene todos los días para incrementar su nivel educativo y por lo tanto sus condiciones de vida, condiciones, calidad de vida, que pueda acceder a un mejor trabajo, ser promovido en donde está, en su empleo y seguir estudiando, incluso abrirse a la oportunidad de seguir estudiando también ya licenciaturas en línea que son gratuitas.
\end{abstract}

En este mismo sentido es considerada la atención diferencia por parte de los docentes como un elemento fundamental dado el apoyo y seguimiento que se le debe dar al estudiante en aras de que logre sus objetivos propuestos durante el curso y de este modo potenciar su desarrollo, por ello: "los profesores prestan una atención muy particular y una atención presencial como apoyo a la tecnología, no se deja solo a la tecnología como responsable de la educación".

\subsection{Estudiantes}

El estudiante seleccionado y su mamá identificaron dos aspectos en los que contribuye a su criterio el BEL al desarrollo social: la motivación y la flexibilidad. Discutiremos en este apartado el que no fue identificado por otros actores.

El plantea que la motivación por parte de familiares y docentes es un elemento fundamental para la culminación de sus estudios, en este sentido plantea: "ya me había desanimado un poco en la cuestión de que no había yo encontrado oportunidad de estudiar, entonces yo ya lo había descartado por completo".

Contrasta con su experiencia anterior al BEL la motivación que recibió ya no solo por su familia sino por docentes y directivos de forma tal que le permitieron culminar sus estudios en el mismo:

fueron como que dándome el ánimo para ir haciendo que mi estancia en BEL pues fuera lo más fácil posible, ¿no? Implementaron programas, de hecho en la sala de BEL se adaptó una computadora en la cual yo podía ir y usarla con programas alternativos los cuáles pues ayudaron mucho para que el desempeño y mi capacidad no se viera 
mermada o limitada en algunos aspectos del uso de la computadora. Entonces ese ánimo que me daban, esa motivación de estarme ayudando siempre pues fue como que el plus, igual pues mi mamá aquí en mi casa dándome ánimo para concluir pues igual fue parte fundamental.

Lo anterior evidencia, cómo el BEL al dar seguimiento a los estudiantes según sus necesidades específicas favorece la culminación de este nivel de enseñanza permitiéndole a los jóvenes desarrollar las capacidades necesarias que atendiendo a Nussbaum (2002) necesita el ser humano para su desarrollo (Nussbaum, 2002)

\title{
3.4. Directivos y docentes
}

Los directivos y docentes coincidieron en identificar la innovación educativa como elemento que se debe contemplar en la enseñanza en línea para contribuir al desarrollo social, evidenciándose las capacidades de "emociones", "razón práctica" y "afiliación" abordadas por Nussbaum (2002), dado que esta permite que el proceso de enseñanza aprendizaje se pueda desarrollar de forma más dinámica, con mayor interacción entre los alumnos y motivadora pues plantean que:

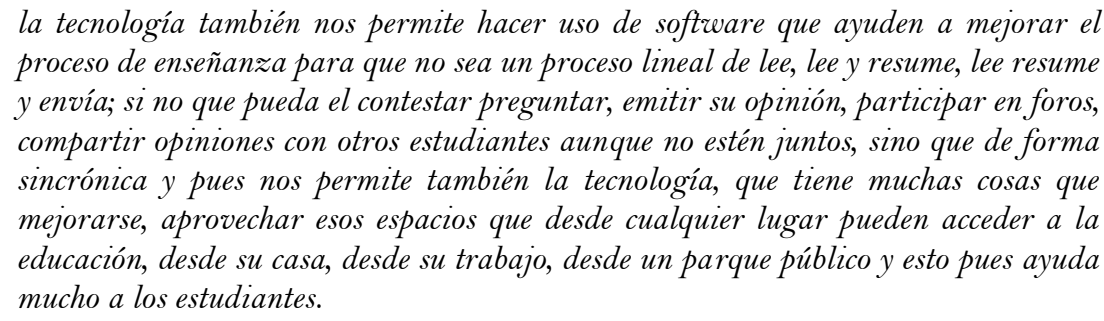

De este modo igual dejan en evidencia la necesidad de contextualizar la enseñanza a las necesidades de los estudiantes de modo que el aprendizaje les sea más ameno y de así disminuir los casos de abandono y deserción escolar pues

\begin{abstract}
hoy en día los estudiantes pues ya vienen formados con estas herramientas tecnológicas, son nativos digitales y bueno pues para que logremos que aprendan pues de mejor manera, pues hay que ponernos en su mismo contexto y hay que aprovechar esas herramientas para potenciar el aprendizaje de los estudiantes.
\end{abstract}

\subsection{Directivos, docentes y estudiante}

Un elemento reiterativo en el discurso de los tres actores participantes fue el de la flexibilidad en cuanto a edad, diversidad funcional (visual, auditiva, motriz) y situación socioeconómica dado que consideran que esta modalidad de enseñanza ha permitido “acercar a más personas a la educación o la capacitación”.

En este aspecto se muestra la contribución del BEL a capacidades de Nussbaum (2002), como la de "vida", "integridad corporal", "afiliación" y "control del propio entorno" dado que declaran que esta propia flexibilidad permite a aquellas personas que no les ha sido posible continuar estudios en enseñanza tradicional retomarlos pues consideran que

\begin{abstract}
la tecnología pues es una herramienta muy importante para poder lograr este propósito de brindar educación de calidad a grupos que no pueden, grupos o personas que no pueden acudir a un centro educativo con un horario establecido, con calendario definido. Entonces me parece que la parte de la educación en línea a distancia pues con estas herramientas tecnológicas permite lograr pues que la universidad pueda llegar a otros rincones y brindar la educación que necesitan pues estos grupos.
\end{abstract}

Profundizando un poco más en las características de este grupo de personas que atiende el BEL plantean: 
gente que nunca tuvo la oportunidad, adulta mayor, adultos mayores que no tuvieron la oportunidad en su momento para cursar estudios de bachillerato y bueno, finalmente deciden dar ese paso. Entonces son diferentes situaciones, condiciones entonces no es el típico estudiante de 18 años, perdón de 15 años, de 15 a 18 años, sino que hay una gama muy diversa pues de edades, de condiciones socioeconómicas, de condiciones personales y eso pues finalmente brinda una mayor flexibilidad para poder lograr esa educación.

En cuanto a la edad, el BEL "tiene en un $60 \%$ poblaciones que no son las típicas en edad para cursar los estudios de bachillerato". Explican que para brindar el acceso educativo a las personas independientemente de su edad deben prestar atención a sus necesidades individuales, por ejemplo "los adultos a los que se les ha dificultado un poco el uso de la tecnología pues aquí que se les apoya para que pueden familiarizarse con la computadora, perderle el miedo y ya ellos seguir".

El estudiante cuenta como el tema de la edad constituyó un factor que frenó la continuación de sus estudios y es esa una de las razones por las que ingresó al BEL "en las demás preparatorias por la edad que yo ya presentaba cuando decidí iniciar" y "las preparatorias ya no me aceptaban a esta edad para iniciar desde un inicio"

Por ello considera que el hecho de que el BEL "abre su campo a varias personas ¿no? no limita su campo de acción a un solo sector, sino que pues es un programa que está abierto a varios sectores de todas las edades. Ese creo que es un muy buen punto a su favor"

En cuanto a diversidad funcional, muestras su experiencia en el trabajo e inclusión de personas con discapacidad visual, motriz y auditiva mostrando algunas adecuaciones y adaptaciones para permitirles una total integración al sistema educativo, al respecto nos cuentan plantean:

Considero que las personas que se han acercado con alguna discapacidad visual, motriz, principalmente o auditiva, pueden satisfacer esa necesidad educativa a través de la tecnología. Ellos tienen su computadora y no necesitan a lo mejor transportarse porque están en silla de ruedas, no necesitan venir hasta acá. Los estudiantes que hemos atendido con alguna discapacidad visual pues se ha instalado un software, en una computadora, específicamente para un estudiante que teníamos con una discapacidad visual muy... o sea no veía, tenía como un 30\% de visión, el software le leía incluso la misma plataforma puede y tiene esta lectura de voz, hay que actualizar unos plug-ins.

De forma específica en lo Visual cuentan su experiencia de cómo "buscamos a lo mejor programas para acercárselos a ellos que les puedan servir”. El estudiante evidencia lo complicado que resulta el enfrentarse a la enseñanza con algún problema de discapacidad, desde su experiencia visual, al respecto nos dice:

$$
\begin{aligned}
& \text { no voy a negar que cuando entre al bachillerato si me desanimó mucho la situación de } \\
& \text { que era en línea que yo pensé que iba a ser un poco más light en la cuestión del estudio. } \\
& \text { Si pensé que iba a ser mucho más fácil entrar ya de lleno al sistema y darme cuenta } \\
& \text { que realmente no era sencillo por ciertos problemas de disminución visual que tengo, } \\
& \text { pues si fue como que un desánimo. }
\end{aligned}
$$

Por lo que en el intento de profundizar en las acciones para apoyar a estudiantes con estas características conocimos que le son brindadas el acceso a herramientas tecnológicas pues:

le dimos una computadora, una lap que podía hacer y ahí ya estaba instalado, o sea si el necesitaba otro tipo de apoyo venia y hacia uso del centro de cómputo. Pero también la misma plataforma hace la lectura de las presentaciones. Ahorita no sé si en la versión que tiene sigue leyendo, pero cuando él estuvo si se podía leer incluso podía grabar, cuando no podía escribir porque tenía que acercarse mucho podía hacer unas 
grabaciones de su voz a través de otra herramienta que es gratuita, y él podía grabarse ya sea que se grabe y grabe su pantalla o que grabe solamente su voz y mandaba esto a los profesores y así se los calificaban, entonces para los de discapacidad visual.

Referente a la atención e inclusión a personas a la educación con dificultades Auditiva los actores mencionan que "hay personas con discapacidad auditiva pero realmente no hemos necesitado realizar como alguna adecuación mayor para ellos", en este sentido explican que las acciones están encaminadas a:

\begin{abstract}
los videos por ejemplo que nosotros compartimos o que los asesores comparten de routube o de ScreenCas, principalmente de Toutube, se pueden poner subtítulos, automáticamente se pueden poner y el mismo asesor puede editar los subtítulos por si no hace la traducción correcta el Youtube, y las lecturas, pues bueno ayuda mucho a las personas que no tienen audición.
\end{abstract}

En cuanto a las necesidades de personas con discapacidad motriz dejaron en evidencia que la mayor contribución del BEL para permitir su inclusión en la educación es la no presencialidad por lo que al no tener que trasladarse les permite continuar sus estudios.

Se pudo constatar que en cuanto a la situación socioeconómica el BEL contribuye aún más dado que en la experiencia de los actores "ayuda en gran medida a igualar estas oportunidades que tienen los estudiantes que no pueden acceder a la educación presencial, tienen la oportunidad de hacer uso de la tecnología para igualar las condiciones educativas y socioeconómicas que van a poder mejorar" y que tras la experiencia en el BEL y el intercambio con los entrevistados plantean que "es un ahorro por el transporte, por el uniforme, por ciertas cuestiones que se tienen que cubrir"

Otro caso identificado en el que contribuye el BEL es para aquellas personas que "por cuestiones económicas tienen que trabajar, entonces bueno pues pueden utilizar el bachillerato en línea para que en horarios fuera de su trabajo puedan lograr". En la experiencia del estudiante entrevistado cuenta que ya que él se encontraba trabajando "pues algo bueno, porque ya podía dedicarme en las tardes o el tiempo que yo considerara para el estudio.”

En aras de conocer las experiencias laborales del estudiante entrevistado se pudo constatar que el BEL contribuyó a su desarrollo puesto que antes de ingresar al BEL este estuvo en:

Un corporativo de vigilancia donde estuve año y medio y pues realmente llega momentos en los cuales ya ni a mi casa llegaba a descansar en los cuales eran jornadas demasiado largas por un sueldo pues relativamente mínimo, o sea no era un sueldo muy bien remunerado entonces pues si ya me habia fastidiado en ese aspecto, ¿no?

Entonces conocer servicios de vigilancia con situaciones pues no muy buenas pues si era pesado, ¿no? entonces pues ya no quise seguir teniendo esa... ese tipo de vida o ese tipo de trabajos y por eso decidí pues ya iniciar un estudio ya más en forma.

Después de esa experiencia en los inicios de su ingreso al BEL

Estaba en una taquería... un señor que se llama Juan Carlos. Él era mi jefe en ese momento, la taquería pues era... estaba ubicada cerca de Chichí Suárez, entonces pues yo salía a la una de la tarde, entraba a las seis de la mañana y salía a la una de la tarde.

A mediados de sus estudios en el BEL "empiezo a trabajar en el área de limpieza y sigo estudiando mi bachillerato puesto que pues ya era un horario más cómodo y un trabajo un poco más estable”. Ya concluido el BEL este joven comenzó a trabajar en Telcel y se encuentra laborando allí como supervisor ya casi un año. 
En cuanto a continuar sus estudios lo intentó en la CTM donde cursó los dos primeros semestres, en este sentido explica:

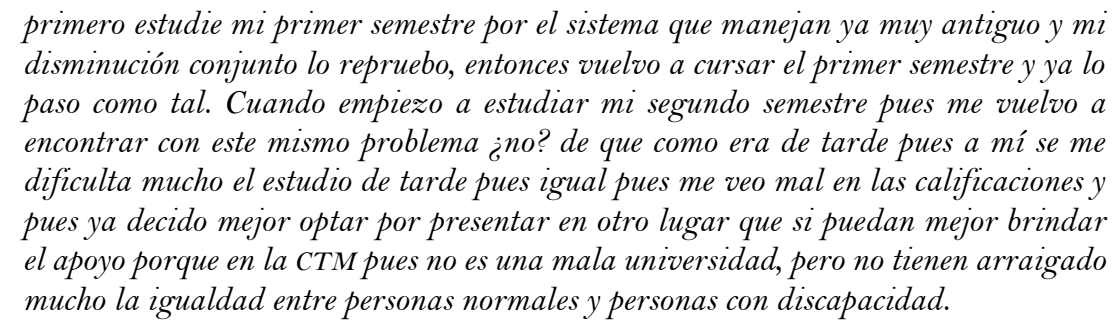

En la actualidad se encuentra preparándose para presentar examen de ingreso a la UADY, al respecto comenta "intentar nuevamente presentar a ver cómo me va esta vez"

\section{Conclusiones}

En la interacción con los actores del BEL, como se muestra en el esquema 1, se encontraron diferentes categorías en cuanto a la función del programa de fomentar una equidad e inclusión educativa que permita un mayor desarrollo social, las cuales fueron analizadas desde la perspectiva de capacidades necesarias para el desarrollo humano según Nussbaum (Boni Aristizábal, 2005).

Atendiendo a los resultados se constató que los actores participantes perciben que el BEL en su propósito de formar bachilleres integrales capaces de contribuir y transformar su entorno acorde a las necesidades sociales, enfrentan retos en cuanto al aspecto económico, de preparación de los docentes para afrontar su papel, de reconocimiento social dado las características del mismo de ser en línea mediado por tecnología y de equidad e inclusión educativa.

Consideran que a pesar de esos retos en su labor cotidiana el programa contribuye al desarrollo social teniendo como principio la igualdad de acceso a la educación independientemente de su edad, procedencia social, género, raza, religión o diversidad funcional; logrando formar alumnos en este nivel de enseñanza acorde a los niveles estandarizados de calidad contribuyendo de este modo a su desarrollo humano.

Fue posible evidenciar a través de la experiencia de los actores involucrados en el BEL que este programa fomenta el desarrollo de las siguientes capacidades de la teoría "vida", "salud corporal", "integridad corporal", "sentidos, imaginación y pensamiento", "emociones", "razón práctica", "afiliación" y "control del propio entorno" planteadas por Nussbaum (2002) pero no se logró constatar el fomento a las capacidades referentes "otras especies" y de "juego"; para lo cual recomendamos se realice un estudio que permita identificar si fomenta estás capacidades o no, de igual modo si existen otras capacidades que fomente dado que Nussbaum platea que pueden existir otras más. 


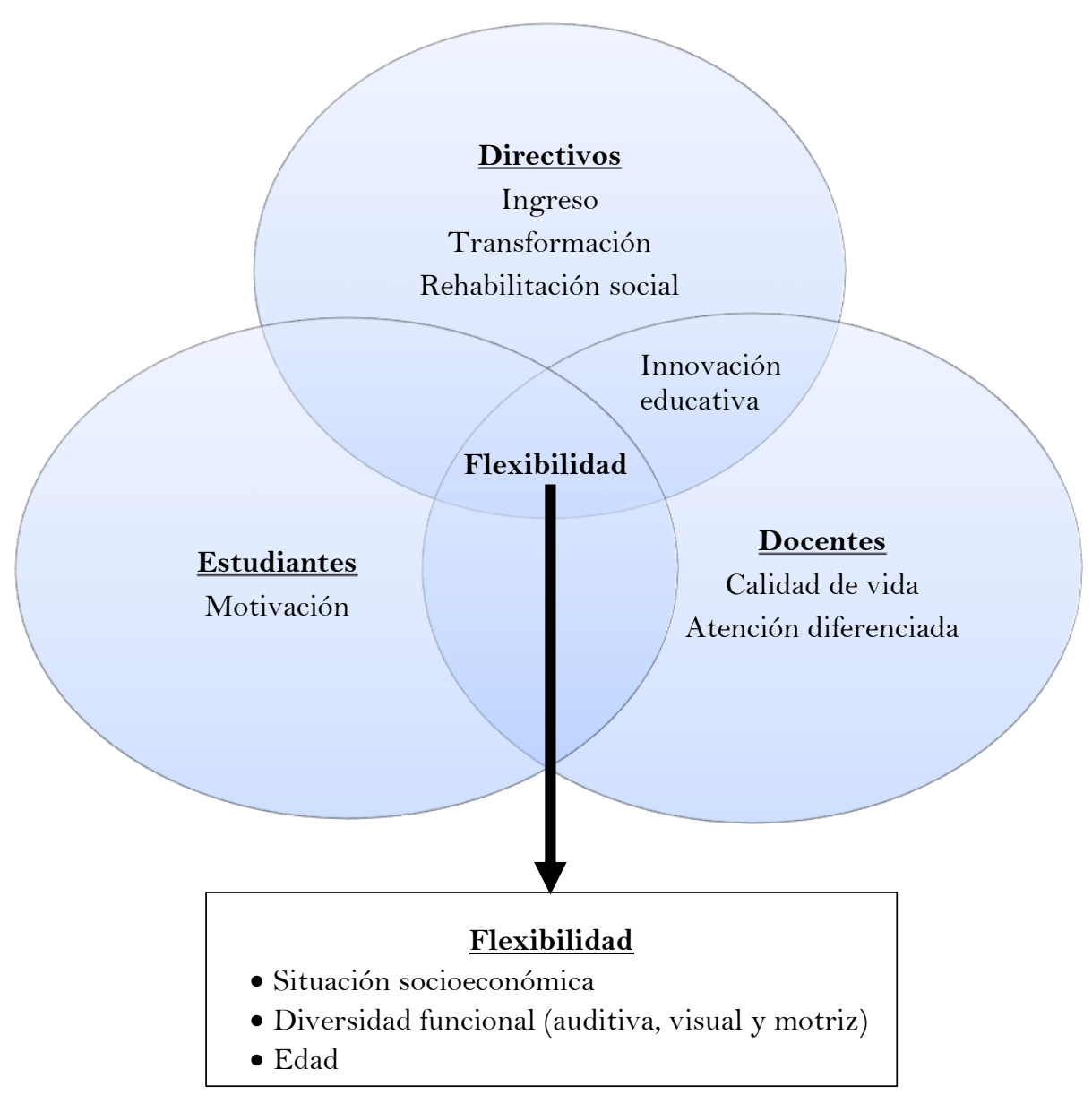

Esquema 1. Categorías encontradas según los actores involucrados de las contribuciones del BEL al desarrollo social

Fuente: Elaboración propia.

Tras la experiencia del alumno graduado de este programa se encontró que el desarrollo que este joven experimentó en el BEL le permitió alcanzar un mejor puesto de trabajo y de este modo su situación económica y calidad de vida se vio transformada, satisfaciendo de este modo su perspectiva de bienestar subjetivo; aunque aún se desconoce si le brindó las herramientas necesarias para continuar estudios superiores, lo cual representa para el estudiante un objetivo a alcanzar y podría ser otro elemento a considerar en estudios posteriores sobre este tema en cuestión.

Este trabajo permitió identificar, que todos los actores involucrados en el BEL coinciden en que la flexibilidad del programa en cuanto a edad, situación socioeconómica y de diversidad funcional es un aspecto que contribuye al desarrollo social, lo cual argumentamos contrastando desde la teoría de las capacidades de Nussbaum. Sin embargo, es pertinente la realización de trabajos futuros que permitan conocer a profundidad esta contribución y examinar con mayor profundidad cuestiones críticas que emergen de los datos; en especial, la forma en que el programa tiene en cuenta las necesidades de un grupo diverso de estudiantes en los procesos de su implementación. Por ejemplo: la tutoría, el seguimiento a los estudiantes, el concepto de buena docencia y su evaluación desde una perspectiva de equidad e inclusión. 


\section{Referencias}

ANUIES. (2015). Entornos virtuales de aprendizaje: Estado del conocimiento en el período 2002-2011. Ciudad de México: Consejo Mexicano de Investigación Educativa.

Badilla, L. (2006). Fundamentos del paradigma cualitativo en la investigación educativa. Revista de Ciencias del Ejercicio y la Salud, 4(1), 42-51.

World Bank. (1994). Higher Education. Washington, D.C.: World Bank.

Boni Aristizábal, A. (2005). La educación para el desarrollo en la enseñanza universitaria como una estrategia de la cooperación orientada al desarrollo humano. Velencia: Universitat de Valencia.

Bracho, T. (2002). Desigualdad social y educación en México. Una perspectiva sociológica. Educar, 29, 31-54.

CONEVAL. (2014). Informe de evaluación de la política de desarrollo social en México en materia de rezago educativo 2011. Ciudad de México: CONEVAL.

Creswell, J. W. (2009). Research Design: Qualitative, Quantitative and Mixed Approaches. Los Angeles, CA: Sage. https://doi.org/10.2307/1523157

De Puelles, M., y Torreblanca, J. I. (1995). Educación, Desarrollo y Equidad Social. Revista Iberoamericana de Educación, 9, 165-189.

Hernández Sampieri, R., y Baptista Lucio, P. (2014). Metodología de la investigación. Ciudad de México: McGraw-Hill-Interamericana.

Hirata, R. (2003). 7 nuevas herramientas para el control de la calidad. Mérida: Kiesen Consultores.

Kent, R. (1995). Debate: dos posturas en el debate internacional sobre la educación superior: el Banco Mundial y la UNESCO. Universidad Futura. Recuperado a partir de http://lanic.utexas.edu/project/lasa95/kent.html

MECD. (2016). Indicadores de la OCDE. Madrid: INEE.

Moreira, M. A. (2002). Investigación en educación en ciencias: métodos cualitativos. Actas del PIDEC, 4, 25-55

Nussbaum, M. (2002). Las Mujeres y el Desarrollo Humano. El Enfoque de las Capacidades. Barcelona: Herder.

Nussbaum, M. (2013). Crear capacidades: propuesta para el desarrollo humano. Barcelona: Paidós.

OCDE. (2015). Panorama de la Educación en México. París: OCDE.

Ordorika, I. (2014). Renovar las políticas de acceso a la educación superior. Revista de la Educación Superior, $\operatorname{XLIII}(170), 5-8$.

Ruiz Olabuenaga, J. I. (2003). Metodología de la investigación cualitativa. Bilbao: Universidad de Deusto.

Sandín, M. P. (2003). Investigación cualitativa en educación. Fundamentos y tradiciones. Madrid: McGrawHill.

Sandoval Casilimas, C. A. (1996). Investigación cualitativa. Bogotá: Arfo.

Schwandt, T. A. (1994). Constructivist, interpretivist approacches to human inquiry. En N. Denzin e Y. Lincol (Eds.), Handbook of qualitative inquiry research (pp. 198-201). Thousand Oaks, CA: Sage.

Sen, A. (1988). The concept of development. En T. N. Chenery, y H. Srinivasan (Eds.), Development economics (pp.1-25). Nueva York, NY: Elsevier.

Sen, A. (2000). Desarrollo y libertad. Desarrollo como Libertad. Buenos Aires: Editorial Planeta.

Stake, R. E. (2007). Investigación con estudio de caso. Madrid: Morata. 
UADY. (2002a). Reglamento interior de las escuelas preparatorias. Recuperado a partir de http://www.transparencia.uady.mx/a9/Documents/reglamentos_int_dep/reglamento interiorPrepa2Vigente.pdf

UADY. (2002b). Estatuto general de la universidad autónoma de Tucatán. Recuperado de http://www.secgral.udg.mx/sites/archivos/normatividad/

UADY. (2011). Reglamento del personal académico. Recuperado de http://www.uady.mx/eventos/files/REGLAMENTOPEDPDEDUCACIONSUPERIOR 2010-2011.pdf.

UADY. (2012a). Modelo educativo para la formación integral. Recuperado de http://www.csems.uady.mx/media/Mefi_EMS_2013.pdf

UADY. (2012b). Programa Educativo de Tipo Medio Superior. Bachillerato en Línea. Recuperado de http://www.dgda.uady.mx/media/docs/mefi_estudiantes.pdf

UADY. (2013). UADY virtual. Plan de Desarrollo 2013-2020. Recuperado de http://es.uadyvirtual.uady.mx/

UADY. (2014). Plan de Desarrollo Institucional. Recuperado de http://www.transparencia.uady.mx/a9/Documents/institucional/pdi2014-2022.pdf

UADY. (2016). Segundo informe de la gestión 2015-2018. Recuperado de http://www.transparencia.uady.mx/a9/Documents/institucional/informeactividades/inf orme-UADY-2015-digital.pdf

UNESCO. (1999). Alfabetización y desarrollo social. Recuperado a partir de http://www.unesco.org/education/uie/confintea/pdf/3c_span.pdf

UNESCO. (2000). Marco de Acción de Dakar. París: Unesco. Recuperado a partir de http://unesdoc.unesco.org/images/0012/001211/121147s.pdf

UNESCO. (2008). Un enfoque de la educación para todos basado en los derechos humanos. Nueva York, NY: Unicef. Recuperado a partir de https://www.unicef.org/spanish/publications/index_42104.html

UNESCO. (2015). Educación 2030 Declaración de Incheon y Marco de Acción para la realización del Objetivo de Desarrollo Sostenible 4. Incheon. Recuperado a partir de http://unesdoc.unesco.org/images/0024/002456/245656s.pdf

UNESCO. (2017). Sitio web: Recuperado a partir de http://www.unesco.org/new/es/education/themes/strengthening-educationsystems/early-childhood/access-and-equity/

Uribe, C. (2004). Desarrollo social y bienestar. Universitas Humanística, 30, 11-25.

Várguez, L. A. (2015). Notas preliminares sobre el bienestar subjetivo en sectores populares de Mérida. En G. Angelotti y G. Reyes (Eds.), Desigualdad y vulnerabilidad social. Reflexiones y estudios de caso (pp. 157-183). Mérida, Yucatán: Editorial UADY.

\section{Breve CV de las autoras}

\section{Yeily Delgado Cruz}

Máster en Tecnologías Aplicadas a los Procesos Educativos, en el Centro Regional de Educación Avanzada. Centro Universitario José Antonio Echeverría, La Habana, Cuba. Cuenta con la categoría docente de Asistente, obtenida en la Universidad de las Ciencias Informáticas, Habana, Cuba. Es estudiante del Doctorado Institucional en Ciencias Sociales de la Universidad Autónoma de Yucatán, Yucatán, México. Sus áreas de investigación son: el uso de las tecnologías en los procesos educativos, interdisciplinariedad, evaluación, la estimulación de alumnos talentosos y potencialmente 
talentosos, atención a necesidades educativas especiales. Sus trabajos se han publicado en México y Latinoamérica, donde también ha realizado numerosas presentaciones en congresos y seminarios. ORCID ID: 0000-0002-9182-2848. Email: yeilydc@gmail.com

\section{Edith J. Cisneros-Cohernour}

Doctora en Ciencias (Ph.D.), en las especialidades de Evaluación, Administración, Educación Superior por la Universidad de Illinois en Urbana-Champaign. Es profesor investigador titular de la Facultad de Educación de la Universidad Autónoma de Yucatán, donde también es la Coordinadora de Investigación de ese centro de estudios. Sus áreas de investigación son: evaluación, desarrollo profesional y organizacional y los aspectos éticos en investigación y evaluación. Asimismo, tiene interés en la enseñanza efectiva de niños de diversa herencia cultural y étnica. Sus trabajos se han publicado en USA, Europa, México y Latinoamérica, donde también ha realizado numerosas presentaciones en congresos y seminarios. ORCID ID: O000-0003-2319-1519. Email: ecohernour@gmail.com 